\title{
AUTOCONCEPTO ACADÉMICO Y TOMA DE DECISIONES EN EL ALUMNADO DE BACHILLERATO
}

\author{
ACADEMIC SELF-CONCEPT AND DECISION MAKING OF SECONDARY \\ SCHOOL STUDENTS
}

\author{
Lidia E. Santana Vega*, Luis A. Feliciano García**, Ana B. Jiménez Llanos*** \\ Facultad de Educación. Universidad de La Laguna
}

\begin{abstract}
RESUMEN
Este trabajo analiza las relaciones entre el autoconcepto académico y el autoconcepto académico percibido con la toma de decisiones de los alumnos de Bachillerato. En la investigación participaron 454 alumnos. El instrumento utilizado fue un cuestionario en el que se incluyó una adaptación para el Bachillerato de las escalas "Qué Opinas de ti Mismo", "Qué Opinan Tus Padres de Ti" y "Qué Opinan Tus profesores de Ti” de Brookover, Erickson y Joiner (1967). Los análisis estadísticos muestran altas correlaciones entre las variables analizadas. Los alumnos que optan por la Universidad: a) valoran más favorablemente su faceta académica; b) perciben en sus padres y en el profesorado una valoración más positiva de su rendimiento en Bachillerato; c) adoptan una actitud más responsable hacia sus estudios que quienes eligen otra opción. Los resultados aportan interesantes sugerencias para generar estrategias de intervención en los procesos de toma de decisiones académico-laborales.
\end{abstract}

Palabras claves: Autoconcepto académico, autoconcepto académico percibido, toma de decisiones, Bachillerato.

\section{ABSTRACT}

This article shows the relationship between academic self-concept, perceived academic self-concept, and decision making in Secondary Education. 454 students take part in this research. The instrument

* Lidia E. Santana Vega es Catedrática de la Universidad de La Laguna (Tenerife). Licenciada en Psicología y Doctora en Filosofía y Ciencias de la Educación. Directora del master universitario Educar y Orientar para la Transición Sociolaboral. Coordinadora del Grupo de Investigación en Orientación Educativa y Sociolaboral(GIOES).1santana@ull.es

** Luis A. Feliciano García es profesor Titular de Universidad. Miembro del Grupo de Investigación en Orientación Educativa y Sociolaboral (GIOES).

*** Ana B. Jiménez Llanos es profesora Contratada Doctora. Miembro del Grupo de Investigación en Orientación Educativa y Sociolaboral (GIOES). 
used in this article was a questionnaire containing an adaptation "Self-Concept of Ability General Scale", "Perceived Parental Evaluation of Ability Scale" and "Perceived Teacher's Evaluations of Ability Scale" from Brookover, Erickson y Joiner (1967). The statistical analysis shows high correlations between analysed variables. The students who choose the University: a) they value their academic years; b) they become aware a good impression about their parents and teachers and about their high school achievement; c) they adopt a more responsible attitude towards their future studies, from those who choose another option. The results provide interesting suggestions to generate intervention strategies in the academic and working decision making process.

Key words: academic self-concept, perceived academic self-concept, decision making, Secondary Education.

\section{Introducción}

Las conductas de las personas se hallan mediatizadas por las interacciones que éstas mantienen con otros sujetos en su medio social. Dentro de dicho contexto, los individuos se percatan de que quienes les rodean reaccionan ante su forma de actuar, convirtiéndose en un objeto de reflexión para sí mismos desde el momento en que asumen las reacciones de los demás hacia ellos (Hargreaves, 1979).

Esta capacidad para asumir el punto de vista de los demás y para considerarse como objeto de reflexión da lugar a la aparición de una serie de opiniones sobre uno mismo con las que los sujetos elaboran su autoconcepto; constructo que hace referencia al conjunto de conocimientos, percepciones, sentimientos y actitudes que las personas tienen sobre sí misma. El autoconcepto no sólo determina lo que un sujeto piensa que es, sino también lo que cree que puede llegar a ser (Burns, 1982).

En la explicación del pensamiento autorreferente se ha empleado constructos como el de autoeficiacia y el de autoconcepto. No obstante, entre ambos existen diferencias: 1) el autoconcepto es una valoración global, mientras que la autoeficacia se refiere al juicio de las personas sobre sus capacidades para realizar con éxito tareas concretas (Bandura, 1987); 2) el autoconcepto incorpora todas las formas de autoconocimiento y de sentimientos de evaluación, la autoeficacia se centra en el logro de una tarea particular.

Una de las funciones más importantes del autoconcepto sería la de orientar lo que la persona tiende a ser en respuesta a la evaluación de sí misma. Este aspecto conductual del autoconcepto adquiere una especial relevancia, ya que supone que la elección de un determinado modo de comportamiento ante unas circunstancias específicas estaría, en parte, condicionada por lo que la persona llega a creer que es capaz de hacer (González-Pienda y cols., 1997).

El autoconcepto no constituye una obra estrictamente personal. Para su génesis es fundamental desarrollar la habilidad de percibir e interpretar las actitudes que nos comunican "los otros" en el proceso de interacción social (Amezcua y Fernández, 2000; Markus, Smith y Moreland, 1985; Rosemberg, 1973). El efecto que "los otros" ejercen sobre el autoconcepto de un sujeto será más importante cuando las actitudes asumidas por éste provengan de personas significativas, es decir, aquéllas con las que se halle más comprometido emocionalmente (Broc, 2000; Burns, 1982; González-Pienda y cols., 1997). 
El autoconcepto percibido es la aprehensión de lo que los demás piensan de nosotros (¿cómo creo que me ven y me valoran las personas que son importantes para mí?). Los padres son los "otros significativos" más relevantes para la formación del autoconcepto en la infancia (Alonso y Román, 2005; Bernardo y cols., 2002; García Caneiro, 2003; Santana Vega, 2007). A medida que el individuo empieza a ampliar su marco relacional, aparecen "otros significativos" que toman parte en la formación del autoconcepto. El alumnado en el decurso de la escolarización obligatoria adquiere nuevas imágenes sobre sí mismo (como amigo, compañero, alumno) mediante los intercambios con sus profesores, con sus compañeros de clase, con sus amigos, etc. (Amezcua y Fernández, 2000; Burns, 1982; Machargo, 1991). Si bien el grupo de iguales y los profesores adquieren una relevancia creciente como "otros significativos", la familia no pierda importancia; todos contribuyen, en mayor o menor medida, al enriquecimiento y desarrollo del autoconcepto (Broc, 2000; García Caneiro, 2003).

El autoconcepto académico es un componente más del autoconcepto general de la persona, constituyendo una fuente de motivación que influye directa y significativamente sobre los logros y las expectativas escolares del alumnado (González-Pienda y cols., 1997): quienes perciben una valoración negativa de su rendimiento presentan un bajo autoconcepto académico, se sienten incapaces de superar los problemas escolares y experimentan falta de confianza en sus posibilidades para alcanzar los objetivos propuestos. En otras palabras, disponen de menos recursos cognitivos y motivacionales que los alumnos con autoconcepto positivo (Núñez y cols., 1998).

A lo largo de las últimas décadas, los estudios sobre el autoconcepto académico se han incrementado, evidenciando su importancia para el aprendizaje y para la mejora del rendimiento escolar (Albiñana, Doménech y Clemente, 1997; Amezcua y Fernández, 2000; Amezcua y Pichardo, 2000; Broc, 2000; Feliciano, 1990; González-Pienda y cols., 2000; Moreano, 2005; Rodríguez Espinar, 1982; Valle y cols., 1999).

El autoconcepto académico, en la medida que incide sobre las expectativas de los alumnos (lo que creen que pueden llegar a ser), posee una particular importancia para la toma de decisiones, especialmente en la etapa de la Educación Secundaria, dada su relevancia para fraguar los itinerarios vitales. El alumnado debe adquirir una conceptualización realista y positiva de sí mismo, en relación con sus valores, metas, intereses y habilidades, para propiciar su madurez vocacional y facilitar la futura toma de decisiones académica y profesional (Carbonero y Lucas, 1999; Ocampo, Ferro y Ojea, 2001; Lozano y Repetto, 2007).

El objetivo de este estudio es analizar las relaciones entre el autoconcepto académico, el autoconcepto académico percibido y las decisiones académico-laborales del alumnado de Bachillerato. Asimismo perseguimos determinar si existen diferencias entre las autoimágenes de quienes desean cursar estudios universitarios y los que eligen otra alternativa académico-laboral. En concreto, nos planteamos los siguientes interrogantes:

a) ¿Existen diferencias entre el autoconcepto académico del alumnado que decide realizar estudios universitarios y el que opta por otra alternativa laboral o formativa?

b) ¿Existen diferencias entre el autoconcepto académico percibido del alumnado que piensa cursar estudios en la Universidad y el que opta por otra alternativa académico-laboral? 
c) ¿Existen diferencias entre las opiniones del alumnado que opta por los estudios universitarios y el que opta por otra alternativa laboral o formativa respecto al modo de abordar el trabajo académico y al valor de los estudios?

\section{Método}

\section{Sujetos}

En la investigación participaron 454 sujetos que cursaban estudios de Bachillerato en tres centros de la Comunidad Autónoma Canaria. Dichos centros tenían un interés común por facilitar la reflexión y mejorar el proceso de toma de decisiones de sus alumnos, lo que les llevó a participar en el proyecto de investigación "La transición académica y sociolaboral de los jóvenes: una propuesta de actuación para el Bachillerato". Los tres IES eran públicos y se ubicaban en las zonas de mayor concentración urbana de Canarias: S/C de Tenerife y Las Palmas de Gran Canaria; perteneciendo dos de ellos a la zona centro de dichas capitales y el tercero a una zona periferico-urbana de rápido crecimiento demográfico en la isla de Tenerife.

Un $60,6 \%$ del alumnado estudiaba $1^{\circ}$ de Bachillerato, mientras que el 39,4\% cursaba $2^{\circ}$. Por sexo, el $44,8 \%$ eran varones y el $55,2 \%$ mujeres. En cuanto a la modalidad cursada, el $48,5 \%$ estaba matriculado en Humanidades y Ciencias Sociales, el 32,1\% en Ciencias de la Naturaleza y de la Salud y el 19,4\% en Tecnología.

\section{Instrumento}

El Grupo de Investigación en Orientación Educativa y Sociolaboral (GIOES) diseñó un cuestionario formado por 12 preguntas de elección múltiple en las que se analizaba las siguientes dimensiones: a) Características académicas; b) Expectativas académico-laborales; c) Organización del trabajo personal; d) Apoyo familiar en los estudios; e) Importancia de las opiniones de otras personas para la toma de decisiones; f) Expectativas paternas y género; g) Valoración de los estudios de Bachillerato. Además, este cuestionario contenía una escala dirigidas a medir el autoconcepto académico (A.Ac.) y dos escalas para examinar el autoconcepto académico percibido (A.Ac. percibido) (ver Anexo). En concreto:

a) Para analizar el A.Ac., nuestro grupo de investigación realizó una adaptación para el Bachillerato de la versión de Rodríguez Espinar (1982) de la Escala “¿Qué Opinas de Ti Mismo?” de Brookover, Erickson y Joiner (1967). Dicha escala contiene 7 ítems (con 5 alternativas puntuables de 1 a 5) que analizan la valoración que el alumnado hace de su faceta académica. En nuestro estudio obtuvimos un $\alpha$ de Cronbach de 0,80 , lo que pone de manifiesto la alta fiabilidad de esta escala. Para examinar la estructura interna de la escala se empleó el método del Análisis de Componentes Principales. La prueba KMO obtuvo un valor de 0,715 que resulta adecuado para el análisis factorial, y la prueba de esfericidad de Bartlet resultó significativa. $(\mathrm{p}<0,000)$. La extracción de la matriz rotada a través de del método varimax generó dos dimensiones principales que explican el 63,03\% de la varianza total: 1) el primer factor agrupa los elementos relacionados con la 
valoración del rendimiento y la capacidad académica; 2) el segundo factor incluye los elementos que se refieren a las expectativas para realizar/finalizar estudios universitarios.

b) Para analizar el Autoconcepto percibido de padres, se elaboró una adaptación para el Bachillerato de la versión de Rodríguez Espinar (1982) de la Escala “¿Qué Opinan Tus Padres de Ti?"” de Brookover, Erickson y Joiner (1967). Contiene 5 ítems (cada uno con 5 alternativas de respuesta puntuables de 1 a 5) que analizan la percepción del alumnado sobre la valoración que sus padres hacen de su faceta académica. En esta escala obtuvimos un coeficiente $\alpha$ de Cronbach de 0,75. La prueba KMO obtuvo un valor de 0,677 y la prueba de esfericidad de Bartlet resultó significativa $(\mathrm{p}<0,000)$. La extracción de la matriz rotada mediante el método varimax generó dos dimensiones principales las cuales explican el 75,73\% de la varianza total: 1) el primer factor incluye los elementos relacionados con las expectativas percibidas en los padres sobre el rendimiento escolar y la capacidad para terminar el Bachillerato y los estudios en la Universidad; 2) el segundo factor engloba elementos relativos a la valoración percibida sobre el rendimiento y la capacidad para culminar los estudios.

c) Para analizar el Autoconcepto percibido de profesores, se realizó una adaptación para el Bachillerato de la versión de Rodríguez Espinar (1982) de la Escala “¿Qué Opinan Tus Profesores de Ti?"” de Brookover, Erickson y Joiner (1967). Contiene 5 ítems (cada uno con 5 alternativas de respuesta puntuables de 1 a 5) que analizan la percepción del alumnado sobre la valoración que hacen de su faceta académica los profesores considerados importantes. En esta escala obtuvimos un coeficiente $\alpha$ de Cronbach de 0,81 . La prueba KMO obtuvo un valor de 0,742 y la prueba de esfericidad de Bartlet resultó significativa $(\mathrm{p}<0,000)$. La extracción de la matriz rotada se realizó mediante el sistema varimax, que generó dos dimensiones principales, las cuales explican el $77,43 \%$ de la varianza total: 1) el primer factor agrupa los elementos relativos a la valoración del rendimiento y de la capacidad percibida en los profesores; 2) el segundo factor incluye los elementos relativos a las expectativas percibidas sobre la capacidad para terminar el Bachillerato y los estudios universitarios.

\section{Análisis de datos}

El análisis de los datos fue realizado a través del programa SPSS.15 y comprendió el examen de los estadísticos descriptivos para cada una de las variables estudiadas, coeficiente de fiabilidad ( $\alpha$ de Cronbach), análisis de componentes principales, coeficientes de correlación $\left(\chi^{2}\right.$, Contingencia, Pearson) y contrastes de medias para grupos independientes.

\section{Procedimiento}

Los cuestionarios fueron distribuidos en los tres centros a través de sus orientadores, y aplicados en cada grupo de alumnos por sus correspondientes tutores en el horario destinado a las sesiones de tutoría. 


\section{Resultados}

\section{Decisión Académico-Laboral}

Por lo que se refiere al tipo de decisión que el alumnado piensa tomar una vez haya terminado sus estudios de Bachillerato (Figura 1), destaca el hecho de que dos terceras partes del mismo piensan continuar sus estudios en la Universidad (72,2\%); siendo los Ciclos Formativos de Grado Superior la opción elegida por sólo el 14,2\% de los sujetos. El 13,6\% restante se muestra indeciso u opta por otra alternativa.

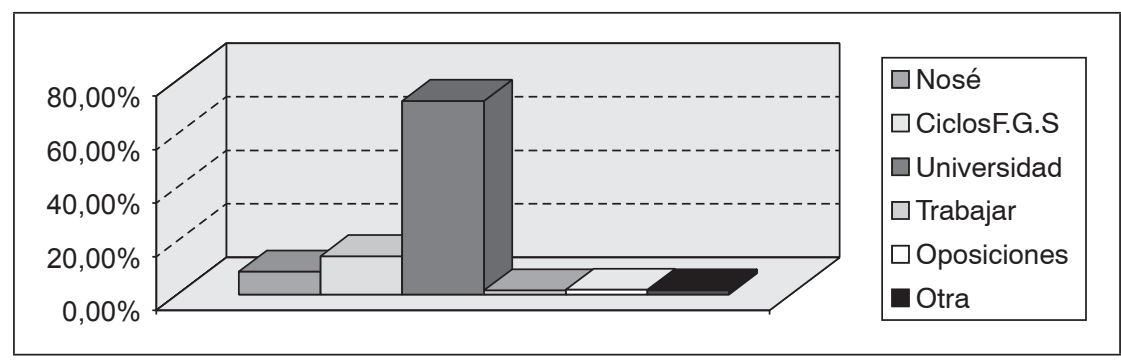

FIGURA 1.

Decisión académico-laboral del alumnado.

\section{Autoconcepto Académico}

La distribución de la valoración del alumnado sobre su trayectoria escolar durante el curso, en función de la decisión que piensa tomar al final del Bachillerato (Tabla 1), revela una tendencia relacional estadísticamente significativa $(\mathrm{p}<0,0001)$.

TABLA 1. "Hasta ahora me ha ido bien en los estudios".

\begin{tabular}{|c|c|c|c|c|}
\hline Decisión & $\begin{array}{c}\text { Total } \\
\text { Desacuerdo }\end{array}$ & $\begin{array}{c}\text { En } \\
\text { Desacuerdo }\end{array}$ & $\begin{array}{c}\text { De } \\
\text { Acuerdo }\end{array}$ & $\begin{array}{c}\text { Total } \\
\text { Acuerdo }\end{array}$ \\
\hline Estudios Universitarios $(\mathrm{N}=326)$ & $1,2 \%$ & $15 \%$ & $42 \%$ & $\mathbf{4 1 , 7 \%}$ \\
\hline Ciclos F.G.S, Otra (N = 124) & $5,6 \%$ & $35,5 \%$ & $45,2 \%$ & $13,7 \%$ \\
\hline
\end{tabular}

$\chi^{2}$ : 46,28; gl: 3; $p<0,0001$; Coeficiente de Contigencia: 0,30; $p<0,0001$.

Conforme a tales resultados, quienes optan por estudiar en la Universidad consideran que "les ha ido bien durante el curso" en mayor porcentaje que quienes toman otras decisiones. Estas opiniones se ven claramente reflejadas en sus puntuaciones de A.Ac. Los resultados del contraste de medias evidencian diferencias significativas $(p<0,0001)$ entre el A.Ac de quienes optan por cursar estudios universitarios y el de los que toman otra decisión (Tabla 2). 
TABLA 2. Autoconcepto Académico y decisión al finalizar el Bachillerato.

\begin{tabular}{|l|c|c|c|c|c|}
\hline \multicolumn{1}{|c|}{ Decisión } & Media & $\begin{array}{c}\text { Desviación } \\
\text { Típica }\end{array}$ & $\mathbf{t}$ & gl & $\begin{array}{c}\text { Sig } \\
\text { (bilateral) }\end{array}$ \\
\hline Estudios Universitarios $(\mathrm{N}=316)$ & 26,15 & 3,46 & & & \\
Ciclos F.G.S. Otra $(\mathrm{N}=118)$ & 21,09 & 4,28 & $-11,500$ & 177,046 & $\mathrm{P}<0,0001$ \\
\hline
\end{tabular}

Estos resultados muestran que la puntuación media de A.Ac. de quienes piensan cursar una carrera universitaria es significativamente superior a la de quienes toman otra decisión.

\section{Autoconcepto Académico percibido}

El contraste de medias entre el A.Ac. percibido de los padres de quienes optan por cursar estudios universitarios y el de los que toman otra decisión pone de manifiesto diferencias significativas entre los dos grupos $(\mathrm{t}=-8,880$; gl: 166,749; $\mathrm{p}<0,0001)$. En este caso, la puntuación media del A.Ac. percibido de los alumnos que piensan cursar una carrera universitaria $(n=320$; Media $=20,51$; DT $=2,45)$ es significativamente superior a la de quienes toman otra decisión $(\mathrm{n}=123$; Media $=17,36$; $\mathrm{DT}=3,63)$.

Otro tanto ocurre con el A.Ac. percibido de los profesores. Los resultados del contraste de medias arrojan diferencias significativas $(t=-9,348$; gl: 178,782; $\mathrm{p}<0,0001)$ entre quienes optan por cursar estudios universitarios y el de los que toman otra decisión. La puntuación media del A.Ac. percibido de quienes piensan cursar una carrera universitaria $(\mathrm{n}=317$; Media $=19,33$; DT $=2,91$ ) es significativamente superior a la de quienes toman otra decisión $(\mathrm{n}=119 ;$ Media $=15,87 ; \mathrm{DT}=3,61)$. La percepción del alumnado sobre la valoración de sus profesores respecto a su trayectoria escolar se halla asociada, por tanto, al tipo de decisión que piensan tomar al final del Bachillerato.

Estos resultados cobran aún mayor relevancia si, tal y como se refleja en la figura 2, un $70 \%$ de los sujetos considera relevante lo que piensan acerca de sus decisiones las personas que les importan.

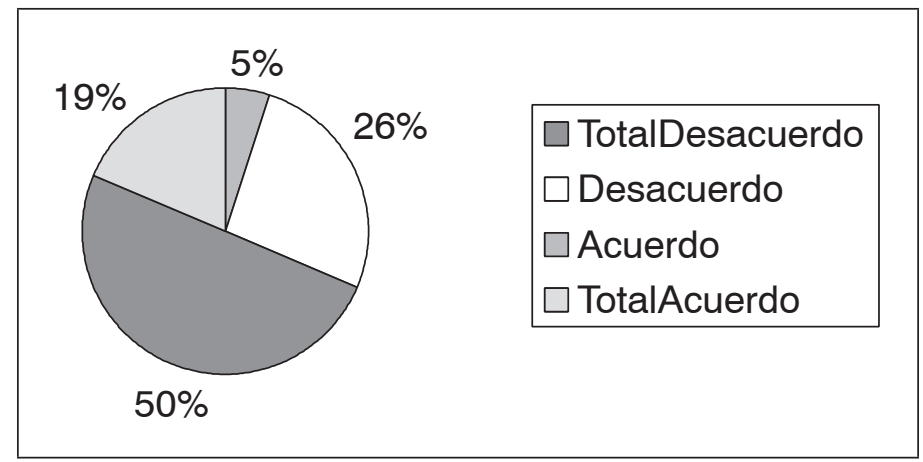

FIGURA 2.

Acuerdo-desacuerdo con la afirmación "Considero relevante lo que piensan sobre mi decisión las personas que me importan". 
Si para la mayoría de los alumnos es relevante la opinión de los "otros significativos" sobre su elección académico-profesional, cabe deducir las consecuencias que en su toma de decisiones tienen las valoraciones percibidas de padres y profesores. No se puede, por tanto, deslindar la toma de decisiones del alumnado del entorno más próximo, rico en informaciones acerca de las posibilidades y limitaciones, tanto en el plano académico como laboral.

\section{Forma de abordar el trabajo académico}

Entre los alumnos que optan por cursar estudios universitarios y quienes eligen otra opción, también hallamos relaciones significativas en lo referente a sus opiniones sobre la forma de abordar el trabajo académico y el valor que éste tiene para ellos. La distribución de las respuestas del alumnado (Tablas 3 a 6) revela tendencias relacionales estadísticamente significativas.

TABLA 3. "Me siento mal si no dedico tiempo a mis estudios".

\begin{tabular}{|c|c|c|c|c|}
\hline Decisión & $\begin{array}{c}\text { Total } \\
\text { Desacuerdo }\end{array}$ & $\begin{array}{c}\text { En } \\
\text { Desacuerdo }\end{array}$ & $\begin{array}{c}\text { De } \\
\text { Acuerdo }\end{array}$ & $\begin{array}{c}\text { Total } \\
\text { Acuerdo }\end{array}$ \\
\hline Estudios Universitarios $(\mathrm{N}=325)$ & $4,6 \%$ & $28,6 \%$ & $\mathbf{4 2 , 8 \%}$ & $\mathbf{2 4 , 0} \%$ \\
\hline Ciclos F.G.S. Otra $(\mathrm{N}=123)$ & $\mathbf{1 0 , 6} \%$ & $\mathbf{3 8 , 9} \%$ & $34,1 \%$ & $15,4 \%$ \\
\hline
\end{tabular}

$\chi^{2}: 13,263 ;$ gl 3; $p<0,004$; Coeficiente de Contigencia: 0,$17 ; p<0,004$.

TABLA 4. "Soy una persona organizada en mis estudios".

\begin{tabular}{|c|c|c|c|c|}
\hline Decisión & $\begin{array}{c}\text { Total } \\
\text { Desacuerdo }\end{array}$ & $\begin{array}{c}\text { En } \\
\text { Desacuerdo }\end{array}$ & $\begin{array}{c}\text { De } \\
\text { Acuerdo }\end{array}$ & $\begin{array}{c}\text { Total } \\
\text { Acuerdo }\end{array}$ \\
\hline Estudios Universitarios $(\mathrm{N}=326)$ & $8,6 \%$ & $36,8 \%$ & $39,6 \%$ & $15,0 \%$ \\
\hline Ciclos F.G.S. Otra (N = 121) & $19,0 \%$ & $43,8 \%$ & $29,8 \%$ & $7,4 \%$ \\
\hline
\end{tabular}

$\chi^{2}:$ 15,737; gl: 3; $p<0,001$; Coeficiente de Contigencia: 0,$18 ; p<0,001$.

TABLA 5. "A veces pienso que tanto estudiar no va conmigo".

\begin{tabular}{|c|c|c|c|c|}
\hline Decisión & $\begin{array}{c}\text { Total } \\
\text { Desacuerdo }\end{array}$ & $\begin{array}{c}\text { En } \\
\text { Desacuerdo }\end{array}$ & $\begin{array}{c}\text { De } \\
\text { Acuerdo }\end{array}$ & $\begin{array}{c}\text { Total } \\
\text { Acuerdo }\end{array}$ \\
\hline Estudios Universitarios $(\mathrm{N}=322)$ & $\mathbf{2 4 , 5 \%}$ & $49,1 \%$ & $27,0 \%$ & $8,4 \%$ \\
\hline Ciclos F.G.S. Otra $(\mathrm{N}=121)$ & $14,9 \%$ & $26,4 \%$ & $38,8 \%$ & $19,8 \%$ \\
\hline
\end{tabular}

$\chi^{2}: 22,314$; gl 3; $p<0,000$; Coeficiente de Contigencia: 0,21; $p<0,000$. 
TABLA 5. "A veces pienso que tanto estudiar no va conmigo".

\begin{tabular}{|l|c|c|c|c|}
\hline \multicolumn{1}{|c|}{ Decisión } & $\begin{array}{c}\text { Total } \\
\text { Desacuerdo }\end{array}$ & $\begin{array}{c}\text { En } \\
\text { Desacuerdo }\end{array}$ & $\begin{array}{c}\text { De } \\
\text { Acuerdo }\end{array}$ & $\begin{array}{c}\text { Total } \\
\text { Acuerdo }\end{array}$ \\
\hline Estudios Universitarios $(\mathrm{N}=324)$ & $\mathbf{2 7 , 5 \%}$ & $\mathbf{4 0 , 1 \%}$ & $24,1 \%$ & $8,3 \%$ \\
\hline Ciclos F.G.S. Otra $(\mathrm{N}=123)$ & $13,8 \%$ & $37,4 \%$ & $\mathbf{3 2 , 5 \%}$ & $\mathbf{1 6 , 3 \%}$ \\
\hline
\end{tabular}

$\chi^{2}: 14,908 ;$ gl 3; $p<0,002$; Coeficiente de Contigencia: 0,18; $p<0,002$.

Así pues, quienes optan por estudiar en la Universidad: "Se sienten mal si no dedican tiempo a sus estudios", "Se consideran organizados en sus estudios", "Se muestran disconformes con la opinión de que estudiar no va con ellos" y "No creen que lo que estudian ahora les servirá de poco en el futuro", en mayor porcentaje que quienes toman otras decisiones.

\section{Conclusiones}

Los resultados del estudio reflejan que la imagen académica del alumnado mediatiza su decisión al finalizar el Bachillerato. A partir de esa autoimagen, los sujetos extraen conclusiones sobre los caminos que se les abren o se les cierran en el plano escolar, lo cual contribuye a configurar su opción académico-profesional. En este sentido, de acuerdo con lo expresado por Ocampo y cols. (2001), el autoconcepto académico es un elemento básico para la futura toma de decisiones de los jóvenes.

Los resultados nos permiten elaborar un perfil de quienes deciden continuar sus estudios en la Universidad, caracterizado por: a) valorar positivamente su faceta académica; b) percibir en sus padres y en el profesorado una valoración positiva de su trayectoria en Bachillerato y de sus posibilidades académicas futuras; c) adoptar una actitud responsable hacia sus estudios, optimizando los aprendizajes.

Quienes no optan por seguir estudios universitarios exhiben un perfil distinto del anterior: a) valoran negativamente su historial académico; b) perciben en sus padres y en sus profesores una valoración negativa de su trayectoria en Bachillerato y de sus posibilidades académicas futuras; c) adoptan una actitud de escepticismo hacia los estudios y su relevancia para su futuro desempeño profesional.

Los resultados concuerdan con los de otras investigaciones realizadas con el alumnado de Educación Primaria y de E.S.O. (Albiñana y cols. 1997; Amezcua y Fernández, 2000; Broc, 2000), donde se muestra que el autoconcepto académico constituye una fuente de motivación potenciadora de los sentimientos de autoeficacia y competencia. Los estudiantes de Bachillerato con bajo autoconcepto manifiestan menos confianza en sus posibilidades académicas que los alumnos con alto autoconcepto. Esta circunstancia adquiere una mayor relevancia en una etapa educativa considerada aún hoy como el visado para continuar los estudios en la Universidad.

La visión negativa de los sujetos sobre su capacidad de logro en los estudios va asociada a la decisión de no cursar estudios universitarios; dicha decisión parece responder más a la circunstancia de tener pobres expectativas académicas, que a un análisis realista y positivo 
de sí mismos, en relación con sus valores, metas, intereses y habilidades. Se debe señalar que mientras hay sujetos con alto autoconcepto académico que deciden trabajar o cursar Ciclos Formativos de Grado Superior, ninguno de los sujetos con bajo autoconcepto prevé cursar estudios universitarios.

Las decisiones del alumnado al término del Bachillerato están en íntima relación con las valoraciones percibidas de padres y profesores sobre su desempeño académico. En consonancia con los resultados obtenidos por Broc (2000), las opiniones de padres y madres siguen siendo relevantes en los alumnos de estas edades para el desarrollo de su autoconcepto académico y, por ende, para la configuración de sus expectativas académico-laborales.

Tal y como señalan autores como Carbonero y Lucas (1999), Santana y cols. (2005), Santana y Feliciano (2006) o Álvarez y cols. (2007), el análisis realista y positivo de sí mismo, de las metas, intereses y habilidades de cada uno ha de formar parte de los programas de orientación educativa y sociolaboral en Educación Primaria, E.S.O y Bachillerato, con el fin de potenciar en los estudiantes sentimientos de competencia y prepararlos para afrontar sus decisiones. Conseguir que el alumnado perciba de manera favorable sus cualidades constituye un objetivo inherente al proceso de enseñanza-aprendizaje en cualquier etapa educativa.

Los resultados de esta investigación vienen a corroborar la necesidad de que el alumnado de Bachillerato ha de incrementar su autoconocimiento en aras a ir potenciando su madurez vocacional. La edad de los estudiantes de esta etapa educativa no es garantía de una preparación adecuada para enfrentarse al reto de la toma de decisiones. La novedad de nuestro trabajo estriba en mostrar precisamente cómo el autoconcepto académico se halla relacionado con el tipo de opción elegida por dichos alumnos, modulando lo que los sujetos creen que pueden llegar a ser. El autoconcepto académico no sólo afecta al rendimiento escolar, como ha sido evidenciado por un extenso número de investigaciones; también es un factor relevante a la hora de elegir la opción adecuada dentro de las coordenadas fijadas por cada sujeto en su proyecto personal de vida. En esta cuestión radica la aportación principal de nuestro trabajo.

Dada la incidencia del profesorado y la familia en el desarrollo del autoconcepto académico, es imprescindible desarrollar estrategias de actuación con los "otros significativos" (Santana Vega y cols. 2003) como parte de la acción orientadora en Bachillerato. No debemos minusvalorar el efecto de las opiniones de los "otros" en la imagen que el alumnado se forma de sí mismo. Percibir una visión positiva de las cualidades personales es, incluso en estas edades, una condición necesaria, aunque no suficiente, para una ajustada valoración de las capacidades. De esta manera el alumnado será capaz de afrontar su decisión al final del Bachillerato sobre la base de un sentimiento de valía personal, no sobre la del pesimismo y el escepticismo.

\section{Agradecimiento}

El trabajo recogido en el presente artículo se ha realizado gracias a la financiación del Proyecto de Investigación PI 2003/131, subvencionado por la Dirección General de Universidades e Investigación del Gobierno de Canarias. 


\section{Referencias bibliográficas}

Albiñana, P., Domenech, F. y Clemente, A. (1997). "Predicción del rendimiento académico en estudiantes de Secundaria a través de los factores de Inteligencia, Socialización, Desadaptación Escolar, Autoconcepto y Personalidad". Revista de Psicología de la Educación, 22, 55-68.

Alonso, J. y Román, J. (2005). "Prácticas educativas familiares y autoestima”. Psicothema, 17 (1), 7682.

Álvarez, M., Bizquerra, R., Espín, V. y Rodríguez, S. (2007). La madurez para la carrera en la Educación Secundaria. Madrid. EOS.

Amezcua, J. y Fernández, E. (2000). "La influencia del autoconcepto en el rendimiento académico". Iberpsicología, 5 (1). http://www.fedap.es/IberPsicologia/iberpsi5-1/amezcua/amezcua.htm.

Amezcua, J. y Pichardo, M. C. (2000). "Diferencias de género en autoconcepto en sujetos adolescentes". Anales de Psicología, 16 (2), 207-214.

Bandura, A, (1987). Pensamiento y acción. Barcelona. Martínez Roca.

Bernardo, A. y cols. (2002). "Inducción parental a la autorregulación, autoconcepto y rendimiento académico". Psicothema, 14 (4), 853-860.

Broc, M. (2000). "Autoconcepto, autoestima y rendimiento académico en alumnos de $4^{\circ}$ de la E.S.O. Implicaciones psicopedagógicas en la orientación y tutoría". Revista de Investigación Educativa, 18 (1), 119-146.

Brookover, W., Erickson, E. y Joiner, L. (1967). Self Concept of Ability and School achievement, III. Cooperative Research Project, $n^{\circ}$ 2831. East Lasing, Michigan State University.

Burns, R. (1982). Self-concept development and education. London. Holt, Rinehart \& Winston.

Carbonero, M. y Lucas, S. (1999). "Desarrollo del autoconcepto y autoeficacia a través de un programa de orientación profesional en Educación Secundaria". Revista de Psicodidáctica, 8, 89-100.

Feliciano, L. (1990). "La Escala ¿Qué opinas de ti mismo? Una vía de aproximación al estudio del autoconcepto académico". Qurriculum, 1, 143-151.

García Caneiro, R. (2003). “Autoconcepto académico y percepción familiar”. Revista Galego-Portuguesa de Psicoloxía e Educación, 9, 359-374.

González-Pienda, J. y cols. (1997). “Autoconcepto, autoestima y aprendizaje escolar”. Psicothema, 9 (2), 271-289.

González-Pienda, y cols. (2000). “Autoconcepto, proceso de atribución causal y metas académicas en niños con y sin dificultades de aprendizaje". Psicothema, 12 (4), 548-556.

Hargreaves, D. (1979). Las relaciones interpersonales en la educación. Madrid. Narcea.

Lozano, S. y Repetto, E. (2007): "Las dificultades en el proceso de decisión vocacional en relación con: el género, el curso académico y los intereses profesionales". Revista de Orientación y Psicopedagogía, 18 (1), pp. 5-16.

Machargo, J. (1991). El profesor y el autoconcepto de sus alumnos: teoría y práctica. Madrid: Escuela Española.

Markus, H., Smith, J. y Moreland, R. (1985). "Role of the self-concept in the perceptions of others". Journal of Personality and Social Psychology, 49, 1494-1512.

Moreano, G. (2005). "Relaciones entre autoconcepto académico, atribuciones de éxito y fracaso y rendimiento académico en escolares preadolescentes". Revista de Psicología, 1, 5-37.

Núñez, J. y cols. (1998). "Estrategias de aprendizaje, autoconcepto y rendimiento académico". Psicothema, 10 (1), 97-109. 
Ocampo, C., Ferro, S. y Ojea, M. (2001). "Psicoloxía vocacional e programas de orientación profesional: aspectos conceptuais básicos". Revista Galego-Portuguesa de Psicoloxía e Educación, 7, $33-42$.

Rodríguez Espinar, S. (1982). Factores del rendimiento escolar. Barcelona. Oikos-Tau.

Rosemberg, M. (1973). La autoimagen del adolescente y la sociedad. Buenos Aires. Paidós.

Santana Vega, L. E. (2007, 2 ${ }^{\mathrm{a}}$ edición). Orientación educativa e intervención psicopedagógica: Cambian los tiempos, cambian las responsabilidades profesionales. Madrid. Pirámide.

Santana Vega, L. E. (Coord.) (2003). Programa de Orientación Educativa y Sociolaboral. Madrid. EOS.

Santana Vega, L. E. y Feliciano, L. (2006). "La construcción de la acción tutorial desde las coordenadas de la investigación colaborativa”. Revista de Educación, 340, 943-971.

Santana Vega, L. E. (directora), Feliciano García, L., Cruz González, A. E., Jiménez Llanos, A. B., Ramos Hernández, H. y Hernández Rivero, V. (2005). "La educación y transición sociolaboral de los jóvenes: una propuesta de actuación para el segundo ciclo de la ESO”. En M.E.C. (Ed.), Premios Nacionales de Investigación Educativa 2004 (pp. 261-290). Madrid. Servicio de Publicaciones del MEC.

Valle, A. y cols. (1999). "Atribuciones causales, autoconcepto y motivación en estudiantes con alto y bajo rendimiento académico". Revista Española de Pedagogía, 57 (214), 525-545.

\section{ANEXO}

Escala: ¿Qué piensan tus padres de ti?

Rodea con un círculo la letra de la respuesta que consideras refleja mejor lo que piensan tus padres de ti.

1. ¿Como crees que valoran tus padres tu capacidad académica en comparación con la de otros alumnos de tu edad?

a. Consideran que estoy entre los mejores.

b. Consideran que estoy por encima de la mayoría.

c. Consideran que estoy entre la mayoría.

d. Consideran que estoy por debajo de la mayoría.

e. Consideran que estoy entre los peores.

2. ¿Crees que tus padres piensan que tienes la capacidad para poder terminar el Bachillerato?
a. Si con seguridad.
b. Si, probablemente.
c. No estoy seguro.
d. Probablemente no.
e. No, con seguridad.

3. ¿Donde crees que tus padres te situarían en la realización de la Prueba de Acceso a la Universidad?

a. Entre los mejores.

b. Por encima de la mayoría.

c. Entre la mayoría. 
d. Por debajo de la Mayoría.

e. Entre los peores.

4. ¿Crees que tus padres piensan que tienes capacidad suficiente para terminar estudios universitarios?
a. Muy probablemente.
b. Con bastante probabilidad.
c. No estoy seguro.
d. Con poca probabilidad.
e. No, con seguridad.

5. ¿Qué tipo de calificaciones consideras que tus padres piensan que eres capaz de obtener?
a. Mayoría de sobresalientes.
b. Mayoría de notables.
c. Mayoría de Bien.
d. Mayoría de suficientes.
e. Mayoría de insuficientes.

Escala: ¿Qué piensan tus profesores de ti?

Piensa ahora en los profesores que creas están más interesados por lo que tú haces en el instituto. Ahora responde a las siguientes preguntas rodeando con un círculo la letra de la respuesta que consideras refleja mejor lo que piensan esos profesores de ti.

1. ¿Como crees que valoran esos profesores tu capacidad académica en comparación con otros alumnos de tu edad?
a. Consideran que estoy entre los mejores.
b. Consideran que estoy por encima de la mayoría.
c. Consideran que estoy entre la mayoría.
d. Consideran que estoy por debajo de la mayoría.
e. Consideran que estoy entre los peores.

2. ¿Crees que esos profesores piensan que tienes capacidad para poder terminar el Bachillerato?
a. Si con seguridad.
b. Si, probablemente.
c. No estoy seguro.
d. Probablemente no.
e. No, con seguridad.

3. ¿Donde crees que esos profesores te situarían en la realización de la Prueba de Acceso a la Universidad?
a. Entre los mejores.
b. Por encima de la mayoría.
c. Entre la mayoría.
d. Por debajo de la Mayoría.
e. Entre los peores. 
4. ¿Crees que esos profesores piensan que eres capaz de terminar estudios universitarios?
a. Muy probablemente.
b. Con bastante probabilidad.
c. No estoy seguro.
d. Con poca probabilidad.
e. No, con seguridad.

5. ¿Qué tipo de calificaciones consideras que esos profesores piensan que eres capaz de obtener?
a. Mayoría de sobresalientes.
b. Mayoría de notables.
c. Mayoría de Bien.
d. Mayoría de suficientes.
e. Mayoría de insuficientes.

\section{Escala: Qué opinas de ti mismo}

Rodea con un círculo la letra de la respuesta que consideras responde mejor a lo que tu opinas sobre ti mismo acerca de tus estudios.

1. ¿Como te clasificarías en cuanto al éxito en tus estudios en comparación con tus mejores amigos?
a. Soy el mejor.
b. Estoy por encima de la mayoría.
c. Soy como la mayoría.
d. Estoy por debajo de la mayoría.
e. Soy el peor.

2. ¿Cómo te clasificarías en cuanto al éxito en tus estudios comparado con el resto de la clase?
a. Estoy entre los mejores.
b. Estoy por encima de la mayoría.
c. Estoy entre la mayoría.
d. Estoy por debajo de la mayoría.
e. Estoy entre los peores.

3. Si hicieras la Prueba de Acceso a la Universidad (PAU), ¿qué tipo de resultados crees que obtendrías?
a. Estaría entre los mejores.
b. Estaría por encima de la mayoría.
c. Estaría entre la mayoría.
d. Estaría por debajo de la Mayoría.
e. Estaría entre los peores.

4. ¿Crees que tienes capacidad para estudiar en la universidad?
a. $\mathrm{Si}$, con toda seguridad.
b. $\mathrm{Si}$, con bastante seguridad. 
c. No estoy seguro.

d. Probablemente no.

e. No, con toda seguridad.

5. ¿Crees que podrías terminas estudios en la universidad?
a. Muy probablemente.
b. Con bastante probabilidad.
c. No estoy seguro.
d. Con poca probabilidad.
e. No, con seguridad.

6. Según tu opinión, ¿cómo crees que es tu trabajo en el instituto?
a. Muy superior al de la mayoría.
b. Superior al de la mayoría.
c. Como el de la mayoría.
d. Inferior al de la mayoría.
e. Muy inferior al de la mayoría.

7. ¿Qué tipo de calificaciones consideras que puedes obtener durante este curso?
a. Mayoría de sobresalientes.
b. Mayoría de notables.
c. Mayoría de Bien.
d. Mayoría de suficientes.
e. Mayoría de insuficientes. 